\title{
Comparación de los resultados a mediano plazo entre la pieloplastia abierta versus laparoscópica en pacientes adultos con obstrucción de la unión ureteropiélica
}

Adrián Ransom-Rodríguez, ${ }_{1}^{1}$ Carlos E. Méndez-Probst, ${ }^{2}$ Bernardo GabilondoPliego, ${ }^{2}$ Elisa Kobashi-Sandoval, ${ }^{1}$ Francisco Rodríguez-Covarrubias ${ }^{2}$

\section{Resumen}

OBJETIVO: Comparar los resultados a mediano plazo entre la pieloplastia abierta versus laparoscópica en pacientes con obstrucción de la unión ureteropiélica primaria o secundaria.

MATERIALES Y MÉTODOS: Estudio descriptivo, retrospectivo, observacional y longitudinal, efectuado en pacientes adultos tratados con pieloplastia abierta o laparoscópica por obstrucción de la unión ureteropiélica, entre enero de 2007 y marzo de 2017. Se analizaron las variables de éxito quirúrgico (cese completo de los síntomas y mejoría de la función renal) y complicaciones posoperatorias con la clasificación de Clavien-Dindo. Se utilizó la prueba de $\chi^{2}$ para comparar las variables cualitativas y la $U$ de Mann-Whitney para las variables independientes con distribución anormal. Se consideró estadísticamente significativo el valor de $p<0.05$.

RESULTADOS: Se registraron 40 pacientes con edad promedio de 39 años y seguimiento de 38 meses (1-180). Los pacientes con pieloplastia laparoscópica tuvieron menor estancia hospitalaria (3.6 \pm 1.4 vs $6.9 \pm$ 2.1 días; $p=0.001)$. La tasa de éxito de pieloplastias laparoscópicas fue de $92 \%$ en pacientes sin tratamiento previo y de $100 \%$ en tratados previamente. Solo se reportaron complicaciones de bajo grado (Clavien-Dindo I y II) en $40 \%$ de los casos con pieloplastia abierta y en $44 \%$ con laparoscopia.

CONCLUSIONES: La pieloplastia abierta y laparoscópica muestran tasas de éxito y resultados similares; por tanto, pueden considerarse técnicas seguras para el tratamiento de la obstrucción ureteropiélica.

PALABRAS CLAVE: Laparoscopia; cirugía; obstrucción de la unión ureteropiélica.

\footnotetext{
${ }^{1}$ Médico pasante de servicio social en Investigación en Urología.

${ }^{2}$ Adscrito al Departamento de Urología.
}

Instituto Nacional de Ciencias Médicas y Nutrición Salvador Zubirán, Ciudad de México.

Recibido: octubre 2017

Aceptado: febrero 2018

Correspondencia

Adrián Ransom Rodríguez

adrianrsm.md@gmail.com

Este artículo debe citarse como

Ransom-Rodríguez A, Méndez-Probst CE, Gabilondo-Pliego B, Kobashi-Sandoval E, RodríguezCovarrubias F. Comparación de los resultados a mediano plazo entre la pieloplastia abierta versus laparoscópica en pacientes adultos con obstrucción de la unión ureteropiélica. Rev Mex Urol. 2018 marzo-abril;78(2):91-97.

DOI: https://doi.org/10.24245/revmexurol.v78i2.1703 
Rev Mex Urol. 2018 March-April;78(2):91-97.

\title{
Comparison of the medium-term results between open pyeloplasty and laparoscopic pyeloplasty in adult patients with ureteropelvic junction obstruction
}

Adrián Ransom-Rodríguez, ${ }^{1}$ Carlos E. Méndez-Probst, ${ }^{2}$ Bernardo GabilondoPliego, ${ }^{2}$ Elisa Kobashi-Sandoval, ${ }^{1}$ Francisco Rodríguez-Covarrubias ${ }^{2}$

\begin{abstract}
OBJECTIVE: To compare the medium-term results between open pyeloplasty and laparoscopic pyeloplasty in patients with primary or secondary ureteropelvic junction obstruction.

MATERIALS AND METHODS: A descriptive, observational, longitudinal, retrospective study was conducted on adult patients that underwent open pyeloplasty or laparoscopic pyeloplasty due to ureteropelvic junction obstruction, within the time frame of January 2007 and March 2017. The variables of surgical success (complete symptom cessation and kidney function improvement) and postoperative complications determined through the Clavien-Dindo classification were analyzed. The qualitative variables were compared using the $\chi^{2}$ test and the independent variables with abnormal distribution were compared using the Mann-Whitney $U$ test. Statistical significance was set at a $\mathrm{p}<0.05$.

RESULTS: Forty patients were registered. Their mean age was 39 years and they had a mean follow-up of 38 months (1-180). The patients that underwent the laparoscopic approach had a shorter hospital stay (3.6 \pm 1.4 days $v s 6.9 \pm 2.1$ days; $p=0.001$ ). The laparoscopic pyeloplasty success rate was $92 \%$ in patients with no prior treatment and $100 \%$ in previously treated patients. Low-grade complications (Clavien-Dindo I and II) presented in $40 \%$ of the open surgery patients and in $44 \%$ of the patients in the laparoscopic group.
\end{abstract}

CONCLUSIONS: Laparoscopic pyeloplasty and open pyeloplasty had similar success rates and outcomes and can be considered safe therapeutic options for the treatment of ureteropelvic junction obstruction.

KEYWORDS: Laparoscopy; Surgery; Ureteropelvic junction obstruction.

\section{ANTECEDENTES}

La obstrucción de la unión ureteropiélica es una alteración que afecta a la población pediátrica y adulta; por tanto, la importancia del diagnóstico y tratamiento tempranos radica en evitar alguna lesión renal permanente y disminuir los síntomas relacionados. 
Aunque su origen suele ser congénito, algunos casos se manifiestan en la etapa adulta, cuando el daño renal es evidente y su corrección quirúrgica está contraindicada. Por lo anterior, la mayor parte de la evidencia científica actual en este campo se enfoca, principalmente, en el estudio de la población pediátrica. Sin embargo, en pacientes con obstrucción adquirida (urolitiasis, neoplasias u obstrucción inflamatoria o posquirúrgica), el deterioro de la función renal es reversible cuando se detecta tempranamente. La incidencia de obstrucción de la unión ureteropiélica es de 1 por cada 1500 adultos. ${ }^{1}$

Las manifestaciones clínicas más frecuentes incluyen: dolor lumbar tipo cólico que puede exacerbarse con el consumo de agua y diuréticos, infecciones recurrentes de la vía urinaria, hidronefrosis, nefrolitiasis, pielonefritis, hematuria, lesión renal aguda o insuficiencia renal crónica; en casos excepcionales suele haber hipertensión arterial sistémica.

El diagnóstico inicial suele establecerse mediante ultrasonido, apoyado con tomografía abdominopélvica contrastada, aunque el estudio de elección es la gammagrafía renal con aplicación de un diurético, pues además de localizar el sitio de la obstrucción es capaz de calcular la tasa de filtrado glomerular mediante radiomarcadores (principalmente mercaptoacetiltriglicina [MAG3]), resultado que puede influir en el protocolo de tratamiento a seguir. Esta técnica se considera diagnóstica cuando la eliminación de la mitad de su dosis infundida (T media) ocurre después de 20 minutos. $^{2}$

El primer procedimiento quirúrgico para el tratamiento de la obstrucción de la unión ureteropiélica fue realizado por Trendelenburg en $1886 .{ }^{3}$ Sin embargo, hasta 1993 Schuessler y su grupo realizaron la primera pieloplastia laparoscópica. ${ }^{4}$ En general, la pieloplastia abierta y la laparoscópica han demostrado resultados similares; no obstante, en la actualidad la pieloplastia laparoscópica (convencional o robótica) se considera el tratamiento de elección, pues provoca menos morbilidad que la técnica abierta. ${ }^{5-7}$

El procedimiento quirúrgico se indica en pacientes con síntomas asociados con la obstrucción, diferencia funcional en la tasa de filtrado glomerular en ambos riñones, nefrolitiasis e hipertensión arterial sistémica. ${ }^{1}$

Existen pocos estudios en México que comparan los resultados de la pieloplastia abierta versus laparoscópica en la reparación de la obstrucción de la unión ureteropiélica en adultos, por lo que hacer una comparación con lo descrito en otros países es difícil. De acuerdo con estos datos, el objetivo de este estudio fue describir y comparar las características preoperatorias, las complicaciones perioperatorias y los resultados a mediano plazo de pacientes con pieloplastia abierta y laparoscópica por obstrucción de la unión ureteropiélica primaria o secundaria.

\section{MATERIALES Y MÉTODOS}

Estudio descriptivo, retrospectivo, observacional y longitudinal, efectuado en pacientes adultos con pieloplastia por diagnóstico de obstrucción de la unión ureteropiélica, atendidos en el Instituto Nacional de Ciencias Médicas y Nutrición Salvador Zubirán, entre enero de 2007 y marzo de 2017.

Se incluyeron a todos los pacientes con diagnóstico de obstrucción de la unión ureteropiélica tratados quirúrgicamente mediante pieloplastia abierta o laparoscópica y se excluyeron los casos con expediente clínico incompleto.

El éxito quirúrgico se definió como la desaparición completa de los síntomas y mejoría de la función renal, determinado mediante gammagrafía renal. Las complicaciones posoperatorias se 
clasificaron según los criterios de Clavien-Dindo en bajo grado (I y II) y alto grado (III, IV y V). ${ }^{8}$

La información obtenida de los expedientes médicos se recabó en una hoja de cálculo (Microsoft Office 2016). Para el análisis estadístico se utilizó el programa Statistical Package for the Social Sciences (SPSS), versión 22 de IBM. Las variables con distribución normal fueron estimadas con media y desviación estándar $( \pm$ DS), mientras que las de distribución anormal con mediana y rango intercuartil, escrito entre corchetes. Se utilizó la prueba de $\chi^{2}$ para comparar las variables cualitativas y la prueba $U$ de Mann-Whitney para las variables independientes con distribución anormal. Se consideró estadísticamente significativo el valor de $\mathrm{p}<0.05$.

\section{RESULTADOS}

Se registraron 40 pacientes con promedio de edad de 39 años. Las características demográficas y manifestaciones clínicas se muestran en el Cuadro 1.

Del total de pacientes, 9 (22.5\%) padecían hipertensión arterial sistémica, 13 (32.5\%) tenían antecedente de litiasis en vías urinarias y 4 (10\%) de pieloplastia previa.
En cuanto a las manifestaciones clínicas, el síntoma más frecuente fue dolor lumbar $(\mathrm{n}=34$; $85 \%)$, seguido de infecciones de vías urinarias de repetición $(n=22 ; 55 \%)$, hematuria $(n=14$; $35 \%)$, náusea y vómito $(n=13 ; 32.5 \%)$.

La principal indicación quirúrgica fue por obstrucción de la unión ureteropiélica aislada: 11 $(73.3 \%)$ pacientes con pieloplastia abierta y 21 (84\%) con laparoscópica. La segunda causa en el grupo de pieloplastia abierta y laparoscópica fue por obstrucción de la unión ureteropiélica y nefrolitiasis $(13.3 \%)$ versus obstrucción de la unión ureteropiélica con hidronefrosis (12\%), respectivamente. En lo que a factor etiológico de la obstrucción ureteropiélica respecta, se identificó el cruce vascular por tomografía abdominal en 7 (17.5\%) casos: 4 en el grupo con laparoscopia y 3 en el de procedimiento abierto. El lado más frecuentemente afectado en ambos grupos fue el izquierdo, con $23(57.5 \%)$ pacientes, mientras que el lado derecho se afectó en 17 (42.5\%) casos.

Se registraron 10 (25\%) pacientes con catéter ureteral doble J antes de la pieloplastia. Estos dispositivos se utilizaron en el posoperatorio en todos los pacientes, por un promedio de 53 días (20-247).

Cuadro 1. Características demográficas y manifestaciones clínicas

\begin{tabular}{|c|c|c|c|c|}
\hline Características & $\begin{array}{l}\text { Total de casos } \\
n=40(\%)\end{array}$ & $\begin{array}{c}\text { Pieloplastia abierta } \\
n=15(\%)\end{array}$ & $\begin{array}{c}\text { Pieloplastia laparoscópica } \\
n=25(\%)\end{array}$ & $\mathbf{p}$ \\
\hline \multicolumn{5}{|l|}{ Sexo } \\
\hline Masculino & $16(40)$ & $9(60)$ & $7(28)$ & $0.048^{*}$ \\
\hline Femenino & $24(60)$ & $6(40)$ & $18(72)$ & \\
\hline Edad (años) & 39 [27.2-56] & $40[25-29]$ & $36[27.5-49.5]$ & $1.000^{* *}$ \\
\hline \multicolumn{5}{|l|}{ Manifestaciones } \\
\hline Dolor lumbar & $34(85)$ & $13(86.7)$ & $21(84)$ & $0.766^{*}$ \\
\hline Hematuria & $14(35)$ & $3(20)$ & $11(44)$ & $0.862 *$ \\
\hline IVU recurrente & $22(55)$ & $8(53.3)$ & $14(56)$ & $0.077^{*}$ \\
\hline Náusea-vómito & $3(32.5)$ & $4(26.7)$ & $9(36)$ & $0.834 *$ \\
\hline \multicolumn{5}{|l|}{ Lateralidad } \\
\hline Derecho & $17(42.5)$ & $7(46.7)$ & $10(40)$ & $0.680^{*}$ \\
\hline Izquierdo & $23(57.5)$ & $8(53.3)$ & $15(60)$ & $0.185^{*}$ \\
\hline
\end{tabular}

IVU: infección de vías urinarias. Prueba de $\chi^{2 *} ; \cup$ de Mann-Whitney**. 
La concentración de creatinina preoperatoria fue de $0.91 \pm 0.26 \mathrm{mg} / \mathrm{dL}$. No se encontraron cambios significativos en la creatinina posoperatoria a seis meses $(0.88 \pm 0.21 \mathrm{mg} / \mathrm{dL}) \mathrm{ni}$ a un año $(0.90 \pm 0.22 \mathrm{mg} / \mathrm{dL})$ del procedimiento $(\mathrm{p}$ $=0.237$ y 0.356 , respectivamente). La tasa de filtrado glomerular no mostró mejoría después del procedimiento quirúrgico $(p=0.941)$.

No se encontró diferencia estadísticamente significativa en cuanto a sangrado en ambos grupos (100 [40-300] vs 50 [40-100] mL en el grupo de pieloplastia abierta y laparoscópica, respectivamente) ( $p=0.233)$. Los pacientes con pieloplastia laparoscópica tuvieron menor estancia hospitalaria $(3.6 \pm 1.4$ vs $6.9 \pm 2.1$ días, respectivamente, $[p=0.001])$. En lo que se refiere a complicaciones posoperatorias, solo se registraron 17 (42.5\%) pacientes de bajo grado (Clavien-Dindo I y II) (Cuadro 2).

De todos los pacientes, 3 (7.5\%) requirieron una nueva pieloplastia por reobstrucción. El seguimiento posoperatorio fue de 38 meses (1-180). Los resultados se describen en el Cuadro 3.

La tasa de éxito global en los pacientes sin pieloplastia previa fue de 95\%, que incluyó a $100 \%$ del grupo de procedimiento abierto y de $92 \%$ del grupo laparoscópico, mientras que en la población con antecedente de pieloplastia se obtuvo una tasa global de éxito de $97.5 \%$, con con falla del tratamiento en un paciente a quien se efectuó pieloplastia abierta.

\section{DISCUSIÓN}

Durante mucho tiempo la pieloplastia abierta representó el tratamiento de elección en pacientes con obstrucción de la unión ureteropiélica, especialmente la pieloplastia desmembrada, descrita por Anderson y Hynes en 1949. ${ }^{9}$ Sin embargo, a pesar de sus ventajas y desventajas (tasa de éxito superior de 95\%, mayor morbilidad relacionada con la cirugía abierta; mayor consumo de analgésicos posoperatorios y mayor tiempo de recuperación), se impulsó la búsqueda de técnicas quirúrgicas menos mórbidas para este tipo de pacientes, como la pieloplastia laparoscópica (convencional o robótica) y la endopielotomía. ${ }^{10,11}$

En un estudio previo reportamos 114 pacientes con obstrucción de la unión ureteropiélica tratados con pieloplastia abierta y endopielotomía. Entre los resultados observamos un promedio de edad similar al del estudio actual (35.5 años) y mayor prevalencia en pacientes masculinos (57\%); sin embargo, el lado más afectado fue el derecho (51.7\%). Asimismo, los resultados funcionales fueron similares a los reportados en esta investigación. ${ }^{12}$

Una desventaja del acceso laparoscópico es la curva de aprendizaje mayor, aunque sus ventajas

Cuadro 2. Complicaciones posquirúrgicas

\begin{tabular}{|c|c|c|c|c|}
\hline $\begin{array}{l}\text { Complicaciones } \\
\text { posquirúrgicas }\end{array}$ & $\begin{array}{c}\text { Total de casos } \\
n=17(\%)\end{array}$ & $\begin{array}{l}\text { Pieloplastia abierta } \\
n=6(\%)\end{array}$ & $\begin{array}{l}\text { Pieloplastia laparoscópica } \\
\qquad n=11(\%)\end{array}$ & p \\
\hline \multicolumn{5}{|l|}{ Clavien-Dindo } \\
\hline I & 7 (17.5) & $1(6.7)$ & $6(24)$ & 0.316 \\
\hline II & $10(25)$ & $5(33.3)$ & $5(20)$ & 0.584 \\
\hline \multicolumn{5}{|l|}{ Clavien-Dindo II } \\
\hline Pielonefritis & $2(5)$ & $1(6.7)$ & $1(4)$ & 0.591 \\
\hline Pielonefritis y urosepsis & $1(2.5)$ & $1(6.7)$ & - & 0.769 \\
\hline Urosepsis & $2(5)$ & $2(13.3)$ & - & 0.826 \\
\hline IVU & $5(12.5)$ & $1(6.7)$ & $4(16)$ & 0.958 \\
\hline
\end{tabular}

IVU: infección de vías urinarias. Prueba de $\chi^{2 *}$ 
Cuadro 3. Resultados a mediano plazo

\begin{tabular}{|c|c|c|c|c|}
\hline Resultados & $\begin{array}{l}\text { Total de casos } \\
n=40(\%)\end{array}$ & $\begin{array}{c}\text { Pieloplastia abierta } \\
n=15(\%)\end{array}$ & $\begin{array}{c}\text { Pieloplastia laparoscópica } \\
n=25(\%)\end{array}$ & $\mathbf{p}$ \\
\hline \multicolumn{5}{|l|}{$\begin{array}{l}\text { Creatinina posoperatoria }(\mathrm{mg} / \mathrm{dL}) \\
6 \text { meses }\end{array}$} \\
\hline 12 meses & $\begin{array}{l}0.88 \pm 0.21 \\
0.90 \pm 0.22\end{array}$ & $\begin{array}{l}0.93 \pm 0.24 \\
0.98 \pm 0.23\end{array}$ & $\begin{array}{l}0.85 \pm 0.18 \\
0.82 \pm 0.20\end{array}$ & $\begin{array}{l}0.237^{*} \\
0.356^{*}\end{array}$ \\
\hline \multicolumn{5}{|l|}{ Gammagrafía renal preoperatorio $(\mathrm{mL} / \mathrm{min})$} \\
\hline TFG global & $\begin{array}{c}98.87 \\
{[0-312.4]}\end{array}$ & $\begin{array}{c}75.35 \\
{[0-119.8]}\end{array}$ & $\begin{array}{c}112.98 \\
{[0-312.4]}\end{array}$ & $0.861^{* *}$ \\
\hline \multicolumn{5}{|l|}{ Gammagrafía renal posoperatoria (mL/min) } \\
\hline TFG global & $\begin{array}{c}78.25 \\
{[10.02-31.87]}\end{array}$ & $\begin{array}{c}74.70 \\
{[53-34.70]}\end{array}$ & $\begin{array}{c}81.40 \\
{[0-133.80]}\end{array}$ & $0.941^{* *}$ \\
\hline
\end{tabular}

TFG: tasa de filtrado glomerular. Prueba de $\chi^{2 *} ; \cup$ de Mann-Whitney**.

radican en menor morbilidad y menos días de estancia hospitalaria posquirúrgicos. No obstante, en este estudio el grupo con pieloplastia laparoscópica tuvo más complicaciones posquirúrgicas, aunque la mayor parte se clasificaron como Clavien-Dindo I, por lo que clínicamente no puede representar un hallazgo significativo; incluso fue un grupo más grande, lo que también puede influir en la prevalencia de complicaciones de los pacientes. El grupo con pieloplastia laparoscópica mostró menor estancia hospitalaria en relación con el de cirugía abierta.

Aunque diversos autores señalan el cruce vascular como una alteración frecuentemente asociada con obstrucción de la unión ureteropiélica, ${ }^{13,14}$ en este estudio solo se registraron 7 (17.5\%) casos: 4 en el grupo con laparoscopia y 3 en el de procedimiento abierto.

A pesar de que la lateralidad no mostró un resultado estadísticamente significativo $(p=0.680)$, el sitio más afectado en ambos grupos fue el izquierdo. El ensayo de Khan y su grupo, en el que evaluaron el tratamiento de la obstrucción de la unión ureteropiélica en adultos, reportó el sitio izquierdo como el lado más frecuentemente afectado. ${ }^{1}$
Maldonado-Valadez y sus colaboradores, en su estudio con 14 casos de obstrucción ureteropiélica tratados con pieloplastia retroperitoneoscópica, reportaron un seguimiento más corto (31.4 meses), estimación de sangrado y tasa de éxito similares al de nuestra casuística (85.7 vs $92.5 \%$, respectivamente). ${ }^{15}$

El estudio de Başataç y su equipo de trabajo, realizado en 56 pacientes con pieloplastia abierta, laparoscópica y asistida con robot, estimaron un sangrado significativamente mayor en el primer grupo, comparado con los otros dos ( $p=0.002$ ); en contraste con este estudio, no se observaron diferencias entre una y otra técnica. ${ }^{10}$

La tasa de éxito de la pieloplastia laparoscópica $(92 \%)$ fue similar a la reportada en la bibliografía internacional (mayor de 95\%); por tanto, la convierte en una técnica efectiva en pacientes mexicanos con obstrucción de la unión ureteropiélica, en centros hospitalarios con recursos y personal adiestrado para realizarla. La relevancia de este hallazgo radica en la reducción de la estancia hospitalaria, posible disminución del riesgo de infecciones intrahospitalarias y gastos médicos, aunque ambas constituyen técnicas quirúrgicas seguras y con tasas de éxito similares. 
La principal fortaleza de este estudio es que representa uno de los pocos ensayos comparativos de pieloplastia abierta versus laparoscópica en México, por lo que sirve de base para sustentar la información científica actual en este campo.

Las limitaciones de nuestro estudio incluyen su naturaleza retrospectiva y el tamaño de muestra pequeño, que impide generalizar los resultados obtenidos en toda la población mexicana, además de no haber realizado el estudio de elección (gammagrama renal) a todos los pacientes, lo que puede suponer un sesgo en los resultados. Puesto que no se trata de un estudio experimental, se limita el establecimiento de la relación causa-efecto o pueden establecerse solamente relaciones.

\section{CONCLUSIONES}

El grupo operado de pieloplastia abierta presentó menos complicaciones postquirúrgicas de bajo grado, mientras que el grupo tratado por vía laparoscópica tuvo una menor estancia hospitalaria en comparación con el grupo abierto. Ambas técnicas quirúrgicas demostraron tasas de éxito y desenlaces similares, por lo cual constituyen métodos seguros para el tratamiento de la obstrucción de la unión ureteropiélica en adultos de nuestra población.

\section{Conflicto de interés}

Los autores declaran no tener ningún conflicto de interés.

\section{Financiamiento}

No se recibió patrocinio para llevar a cabo este estudio.

\section{REFERENCIAS}

1. Khan F, Ahmed K, Lee N, Challacombe B, Khan MS, Dasgupta P. Management of ureteropelvic junction obstruction in adults. Nat Rev Urol 2014;11:629-38.
2. O'Reilly PH, Lawson RS, Shields RA, Testa HJ. Idiopathic hydronephrosis -the diuresis renogram: a new non-invasive method of assessing equivocal pelvioureteral junction obstruction. J Urol 1979;121:153-55.

3. Poulakis V, Witzsch U, Schultheiss D, Rathert P, Becht E. Die Geschichte der operativen Behandlung der Harnleiterabgangsstenose (Pyeloplastik). Der Urol Ausgabe A 2004;43:1544-59.

4. Schuessler WW, Grune MT, Tecuanhuey LV, Preminger GM. Laparoscopic dismembered pyeloplasty. J Urol 1993;150:1795-9.

5. Rogers A, Hasan T. Management of secondary pelviureteric junction obstruction. Ind J Urol 2013;29:294-302.

6. Rassweiler JJ, Subotic S, Feist-Schwenk M, Sugiono $M$, Schulze $M$, Teber $D$, et al. Minimally invasive treatment of ureteropelvic junction obstruction: Long-term experience with an algorithm for laser endopyelotomy and laparoscopic retroperitoneal pyeloplasty. J Urol 2007;177:1000-5.

7. Klingler HC, Remzi M, Janetschek G, Kratzik C, Marberger MJ. Comparison of Open versus Laparoscopic Pyeloplasty Techniques in Treatment of Uretero-Pelvic Junction Obstruction. Eur Urol 2003;44:340-5.

8. Clavien PA, Barkun J, de Oliveira ML, Vauthey JN, Dindo $D$, Schulick RD, et al. The Clavien-Dindo classification of surgical complications: five-year experience. Ann Surg 2009;250:187-196.

9. Patel V. Robot-assisted laparoscopic dismembered pyeloplasty. Urology 2005;66:45-9.

10. Başataç C, Boylu U, Önol FF, Gümüş Eyüp. Comparison of surgical and functional outcomes of open, laparoscopic and robotic pyeloplasty for the treatment of ureteropelvic junction obstruction. Turk J Urol 2014;40:24-30.

11. Huang $Y, W u Y$, Shan $W$, Zeng L, Huang L. An updated meta-analysis of laparoscopic versus open pyeloplasty for ureteropelvic junction obstruction in children. Int J Clin Exp Med 2015;15:4922-31.

12. Castillejos-Molina RA, Rodríguez-Covarrubias F, FeriaBernal G, Gabilondo-Navarro F. Obstrucción ureteropiélica, tratamiento quirúrgico. Gac Med Méx 2006;142:205-8.

13. Janetschek G, Peschel R, Frauscher F. Laparoscopic pyeloplasty. Urol Clin North Am 2000;27:695.

14. Frauscher F, Janetschek G, Klauser A, Peschel R, Halpern EJ, Pallwein L, et al. Laparoscopic pyeloplasty for UPJ obstruction with crossing vessels: contrast-enhanced colour Doppler findings and long-term outcome. Urology 2002;59:500.

15. Maldonado-Valadez R, Badillo-Santoyo MA, Sánchez-Gutiérrez J, Negrete-Pulido O, Manzo-Pérez G, Vanzzini-Guerrero M. Pieloplastia retroperitoneoscópica. Experiencia en el Hospital Regional de Alta Especialidad del Bajío, León, Guanajuato, México. Rev Mex Urol 2014;74(4):204-7. 